\title{
Orbital Stabilization of Underactuated Systems using Virtual Holonomic Constraints and Impulse Controlled Poincaré Maps *
}

\author{
Nilay Kant ${ }^{\mathrm{a}}$, Ranjan Mukherjee ${ }^{\mathrm{a}}$ \\ ${ }^{a}$ Department of Mechanical Engineering, Michigan State University, East Lansing, USA
}

\begin{abstract}
The problem of orbital stabilization of underactuated mechanical systems with one passive degree-of-freedom (DOF) is revisited. Virtual holonomic constraints are enforced using a continuous controller; this results in a dense set of closed orbits on a constraint manifold. A desired orbit is selected on the manifold and a Poincaré section is constructed at a fixed point on the orbit. The corresponding Poincaré map is linearized about the fixed point; this results in a discrete linear time-invariant system. To stabilize the desired orbit, impulsive inputs are applied when the system trajectory crosses the Poincaré section; these inputs can be designed using standard techniques such as LQR. The Impulse Controlled Poincaré Map (ICPM) based control design has lower complexity and computational cost than control designs proposed earlier. The generality of the ICPM approach is demonstrated using the 2-DOF cart-pendulum and the 3-DOF tiptoebot.
\end{abstract}

Key words: Impulsive control, orbital stabilization, Poincaré map, underactuated system, virtual holonomic constraint

\section{Introduction}

For underactuated systems, Virtual Holonomic Constraint (VHC) based control designs have gained popularity due to their conceptual simplicity and applicability to control of repetitive motion; they have been used for gait stabilization in bipeds $[2,6,7,25]$ and trajectory control for systems with open kinematic chains [3,5,14,17,18,20-23]. VHCs parameterize the active joint variables in terms of the passive joint variables and confine system trajectories to a constraint manifold [14]. To enforce the VHC, the constraint manifold has to be stabilized using feedback. Typically, a constraint manifold contains a dense set of periodic orbits and the choice of repetitive motion determines the specific orbit that has to be stabilized. To stabilize biped gaits, for example, Grizzle et.al $[6,25]$ enforced the VHC and periodic loss of energy due to ground-foot interaction was exploited for orbital stabilization.

A special class of underactuated systems are those with one passive DOF. For such systems, Shiriaev and collaborators [5, 20, 23] used VHC to select the desired orbit. For an $n$-DOF system, the $2 n$ dimensional dynamics is linearized about the desired orbit; this results in a $2 n-1$ dimensional system. A periodic Ricatti equation is then solved to design a time-varying controller that stabilizes the orbit. It should be noted that the control designs in $[5,20,23]$ stabilize the orbit

\footnotetext{
* The authors acknowledge the support provided by the National Science Foundation, Grant CMMI-1462118.

Corresponding author Ranjan Mukherjee.

Email addresses: kantnila@egr.msu.edu (Nilay Kant), mukherji@egr.msu.edu (Ranjan Mukherjee).
}

but do not enforce the VHC. A control scheme that enforces the VHC and simultaneously stabilizes the orbit was recently proposed in [17]. The key idea is that the VHC is made time-varying using a scalar parameter which is controlled via feedback. The stabilization problem involves solving a periodic Ricatti equation; however, unlike [5, 20,23], where the dimension of the system is $2 n-1$, the dimension of the system in [17] is always three. For systems with more than two DOF, the method in [17] reduces the computational complexity of control implementation. Also, by enforcing the VHC, it improves control over transient characteristics of the trajectory [16]. Similar to $[16,17]$, we propose a control design that enforces the VHC and stabilizes the desired orbit. The control design is comprised of continuous inputs that enforce the VHC and impulsive inputs that exponentially stabilize the orbit. Impulsive inputs have been used for control of underactuated systems $[1,8-11,15]$ and it has been established that such inputs can be implemented in standard hardware using high-gain feedback.

This paper is organized as follows. The system dynamics is presented in section 2 and the results in [14] are utilized to enforce VHC such that the resulting zero dynamics is EulerLagrange. A periodic orbit is selected on the constraint manifold and a method for orbital stabilization is presented in section 3. To stabilize the orbit, a Poincaré section is defined at a point on the orbit and the return map is linearized about the fixed point; this results in a $2 n-1$ dimensional discrete linear time-invariant (LTI) system. To control this system and stabilize the orbit, impulsive inputs are applied when the system trajectory crosses the Poincaré section. The controllability of the orbit can be verified by simply checking the controllability of the linear system. This is simpler than 
the approach in $[5,20,23]$ where controllability is verified numerically along the orbit for most systems. Since the system is LTI, the control design involves constant gains that can be computed off-line. Compared to the methods proposed earlier [5, 17, 20,23], where periodic Ricatti equations have to be solved, our method has lower computational cost and complexity. Since impulsive inputs are used to control the Poincare map, the closed-loop system dynamics can be described by the Impulse Controlled Poincaré Map (ICPM). The simplicity and generality of the ICPM approach to orbital stabilization is demonstrated using the examples of the 2-DOF cart-pendulum in section 4 and the 3-DOF tiptoebot in section 5. Concluding remarks are presented in section 6 .

\section{Problem Formulation}

\subsection{System Dynamics}

Consider an $n$ DOF underactuated system with one passive DOF, where the passive DOF is a revolute joint. Let $q, q \triangleq$ $\left[\begin{array}{ll}q_{1}^{T} & q_{2}\end{array}\right]^{T}$, denote the generalized coordinates, where $q_{1} \in$ $R^{n-1}$ and $q_{2} \in S, S=R$ modulo $2 \pi$, are the coordinates of the active and the passive DOFs. The configuration space of the system is denoted by $\mathbb{Q}^{n}, \mathbb{Q}^{n} \in R^{n-1} \times S$. The Lagrangian of the system can be written as

$$
L(q, \dot{q})=\frac{1}{2} \dot{q}^{T} M(q) \dot{q}+\mathcal{F}(q)
$$

In the equation above, $M(q) \in R^{n \times n}$ denotes the symmetric, positive-definite mass matrix, partitioned as

$$
M(q)=\left[\begin{array}{l|l}
M_{11}(q) & M_{12}(q) \\
\hline M_{12}^{T}(q) & M_{22}(q)
\end{array}\right]
$$

where $M_{11} \in R^{(n-1) \times(n-1)}, M_{22} \in R$ and $\mathcal{F}(q)$ is the potential energy of the system. The Euler-Lagrange equation of motion can be written as follows

$$
\begin{aligned}
& M_{11}(q) \ddot{q}_{1}+M_{12}(q) \ddot{q}_{2}+h_{1}(q, \dot{q})=u \\
& M_{12}^{T}(q) \ddot{q}_{1}+M_{22}(q) \ddot{q}_{2}+h_{2}(q, \dot{q})=0
\end{aligned}
$$

where $u \in R^{n-1}$ is the control input, and $\left[h_{1}^{T}, h_{2}\right]^{T}$ is the vector of Coriolis, centrifugal and gravity forces. In compact form, (1a) and (1b) can be rewritten as

$$
\begin{aligned}
& \ddot{q}_{1}=A(q, \dot{q})+B(q) u \\
& \ddot{q}_{2}=C(q, \dot{q})+D(q) u
\end{aligned}
$$

where,

$$
\begin{aligned}
B(q) & =\left[M_{11}-\left(1 / M_{22}\right) M_{12} M_{12}^{T}\right]^{-1} \\
A(q, \dot{q}) & =\left(1 / M_{22}\right) B(q)\left[M_{12} h_{2}-h_{1} M_{22}\right] \\
D(q) & =-\left(1 / M_{22}\right) M_{12}^{T} B(q) \\
C(q, \dot{q}) & =-\left(1 / M_{22}\right)\left[M_{12}^{T} A(q, \dot{q})+h_{2}\right]
\end{aligned}
$$

Similar to [14], we make the following assumption:

Assumption 1 For some $\bar{q} \triangleq\left[\bar{q}_{1}^{T}, \bar{q}_{2}\right]^{T} \in \mathbb{Q}^{n}$, the mass matrix $M(q)$ and the potential energy $\mathcal{F}(q)$ are even with respect to $\bar{q}$, i.e.,

$$
M(\bar{q}+q)=M(\bar{q}-q), \quad \mathcal{F}(\bar{q}+q)=\mathcal{F}(\bar{q}-q)
$$

\subsection{Imposing Virtual Holonomic Constraints (VHC)}

A holonomic constraint enforced by feedback is referred to as VHC. The current and the next subsection summarizes relevant results from [14]. For a wide class of mechanical systems, a comprehensive discussion on VHC can be found in [14], [16].

A VHC for (1) is described by the relation $\rho(q)=0$ where, $\rho: \mathbb{Q}^{n} \rightarrow R^{n-1}$ is smooth and $\operatorname{rank}\left[J_{q}(\rho)\right]=n-1$ for all $q \in \rho^{-1}(0)$. Here, $J_{q}(\rho)$ is the Jacobian of $\rho$ with respect to $q$. The VHC is said to be stabilizable if there exists a smooth feedback $u_{c}(q, \dot{q})$ that asymptotically stabilizes the set

$$
\mathcal{C}=\left\{(q, \dot{q}): \rho(q)=0, J_{q}(\rho) \dot{q}=0\right\}
$$

The set $\mathcal{C}$, which is referred to as the constraint manifold, is controlled invariant [14]. For the system described by (1), $\mathcal{C}$ is an $(n-1)$ dimensional manifold.

An important goal of this paper is to generate repetitive motion, which can be described by closed orbits. Consequently, $\rho^{-1}(0)$ must be a smooth and closed curve without any selfintersection. The VHC can be described as

$$
\rho(q)=q_{1}-\Phi\left(q_{2}\right)=0
$$

where $\Phi: S \rightarrow R^{n-1}$ is a smooth vector-valued function. The constraint manifold $\mathcal{C}$ in (4) can be expressed as:

$$
\mathcal{C}=\left\{(q, \dot{q}): q_{1}=\Phi\left(q_{2}\right), \dot{q}_{1}=\left[\frac{\partial \Phi}{\partial q_{2}}\right] \dot{q}_{2}\right\}
$$

It should be noted that since $q_{2} \in S, \Phi\left(q_{2}+2 \pi\right)=\Phi\left(q_{2}\right)$ and $\rho^{-1}(0)$ is closed. Following the notion of odd VHC [14], we state another assumption.

Assumption 2 For $\bar{q}$ which satisfies Assumption 1, $\Phi\left(q_{2}\right)$ is odd with respect to $\bar{q}_{2}$, i.e.,

$$
\Phi\left(\bar{q}_{2}+q_{2}\right)=-\Phi\left(\bar{q}_{2}-q_{2}\right)
$$

To stabilize $\mathcal{C}$, we investigate the dynamics of $\rho(q)$; differentiating $\rho(q)$ twice with respect to time, we get

$$
\ddot{\rho}=\ddot{q}_{1}-\left[\frac{\partial \Phi}{\partial q_{2}}\right] \ddot{q}_{2}-\left[\frac{\partial^{2} \Phi}{\partial q_{2}^{2}}\right] \dot{q}_{2}^{2}
$$


Substitution of $\ddot{q}_{1}$ and $\ddot{q}_{2}$ from (2a) and (2b) in (7) yields

$$
\ddot{\rho}=A-\left[\frac{\partial^{2} \Phi}{\partial q_{2}^{2}}\right] \dot{q}_{2}^{2}-\left[\frac{\partial \Phi}{\partial q_{2}}\right] C+\left[B-\left[\frac{\partial \Phi}{\partial q_{2}}\right] D\right] u
$$

The following choice of linearizing control

$$
\begin{aligned}
u_{c}=\left[B-\left[\frac{\partial \Phi}{\partial q_{2}}\right] D\right]^{-1} & {\left[-A+\left[\frac{\partial^{2} \Phi}{\partial q_{2}^{2}}\right] \dot{q}_{2}^{2}\right.} \\
+ & {\left.\left[\frac{\partial \Phi}{\partial q_{2}}\right] C-k_{p} \rho-k_{d} \dot{\rho}\right] }
\end{aligned}
$$

where $k_{p}$ and $k_{d}$ are positive definite matrices, results in

$$
\ddot{\rho}+k_{d} \dot{\rho}+k_{p} \rho=0
$$

This implies that $\lim _{t \rightarrow \infty} \rho(t) \rightarrow 0$ exponentially and $u_{c}$ in (9) stabilizes the VHC in (5). If the initial conditions are chosen such that $\rho(0)=\dot{\rho}(0)=0, u_{c}$ in (9) enforces the VHC and the constraint manifold $\mathcal{C}$ is controlled invariant.

Remark 1 For $u_{c}$ in (9) to be well-defined, the matrix $\left[B-\left(\partial \Phi / \partial q_{2}\right) D\right]$ must be invertible. It can be shown that $\left[B-\left(\partial \Phi / \partial q_{2}\right) D\right]$ is invertible iff $M_{12}^{T}\left(\partial \Phi / \partial q_{2}\right)+M_{22} \neq$ 0 . This is also a necessary and sufficient condition for $\mathcal{C}$ to be stabilizable - see proposition 3.2 of [14].

\subsection{Zero Dynamics and Periodic Orbits}

On the constraint manifold $\mathcal{C}$, the dynamics of the system satisfies $\rho(q) \equiv 0$; this implies

$q_{1}=\Phi\left(q_{2}\right), \quad \dot{q}_{1}=\left[\frac{\partial \Phi}{\partial q_{2}}\right] \dot{q}_{2}, \quad \ddot{q}_{1}=\left[\frac{\partial^{2} \Phi}{\partial q_{2}^{2}}\right] \dot{q}_{2}^{2}+\left[\frac{\partial \Phi}{\partial q_{2}}\right] \ddot{q}_{2}$

Substitution of $q_{1}, \dot{q}_{1}$ and $\ddot{q}_{1}$ from (11) in (1b) provides the zero dynamics, which can be expressed in the following form

$$
\ddot{q}_{2}=\alpha_{1}\left(q_{2}\right)+\alpha_{2}\left(q_{2}\right) \dot{q}_{2}^{2}
$$

It was shown in $[14,20-22]$ that the equation above has an integral of motion of the form

$$
\begin{aligned}
E\left(q_{2}, \dot{q}_{2}\right) & =(1 / 2) \mathcal{M}\left(q_{2}\right) \dot{q}_{2}^{2}+\mathcal{P}\left(q_{2}\right) \\
\mathcal{M}\left(q_{2}\right) & =\exp \left(-2 \int_{0}^{q_{2}} \alpha_{2}(\tau) d \tau\right) \\
\mathcal{P}\left(q_{2}\right) & =-\int_{0}^{q_{2}} \alpha_{1}(\tau) \mathcal{M}(\tau) d \tau
\end{aligned}
$$

where $\mathcal{M}\left(q_{2}\right)$ is the mass and $\mathcal{P}\left(q_{2}\right)$ is the potential energy of the reduced system in (12). Since Assumption 1 and 2 are satisfied, the zero dynamics represents an Euler-Lagrange system with the Lagrangian 1 equal to $(1 / 2) \mathcal{M}\left(q_{2}\right) \dot{q}_{2}^{2}-$ $\mathcal{P}\left(q_{2}\right)$.

The zero dynamics in (12) is similar to the dynamics of a simple pendulum and its qualitative properties can be described by the potential energy $\mathcal{P}\left(q_{2}\right)$. Let $\mathcal{P}_{\text {min }}$ and $\mathcal{P}_{\text {max }}$ denote the minimum and maximum values of $\mathcal{P}$. If an energy level set is denoted by $E\left(q_{2}, \dot{q}_{2}\right)=c$, then $c \in\left(\mathcal{P}_{\min }, \mathcal{P}_{\max }\right)$ corresponds to a periodic orbit where the sign of $\dot{q}_{2}$ changes periodically and $c>\mathcal{P}_{\max }$ corresponds to an orbit where the sign of $\dot{q}_{2}$ does not change [14].

\subsection{Problem Statement}

Since the zero dynamics in (12) has an Euler-Lagrange structure, there cannot exist any non-trivial isolated periodic orbit - this follows from the Poincaré-Lyapunov-LiouvilleArnol'd theorem [14, 16, 19]. A direct implication of this theorem is that the reduced dynamics possesses a dense set of closed orbits, that are unstable. For a desired repetitive motion, the corresponding orbit must be stabilized. Consider the desired closed orbit $\mathcal{O}_{d}$, defined as follows:

$$
\mathcal{O}_{d}=\left\{q, \dot{q} \in \mathcal{C}: E\left(q_{2}, \dot{q}_{2}\right)=c_{d}\right\}, \quad c_{d}>\mathcal{P}_{\min }
$$

Let $x, x \triangleq\left[q^{T}, \dot{q}^{T}\right]^{T}$, denote the states of the system in (1). We define an $\epsilon$-neighborhood of $\mathcal{O}_{d}$ by

$$
\begin{aligned}
& U_{\epsilon}=\left\{x \in \mathbb{Q}^{n} \times R^{n}: \operatorname{dist}\left(x, \mathcal{O}_{d}\right)<\epsilon\right\} \\
& \operatorname{dist}\left(x, \mathcal{O}_{d}\right) \triangleq \inf _{y \in \mathcal{O}_{d}}\|x-y\|
\end{aligned}
$$

We now define stability of the orbit $\mathcal{O}_{d}$ from [12].

Definition 1 The orbit $\mathcal{O}_{d}$ in (14) is

- stable, if for every $\epsilon>0$, there is a $\delta>0$ such that $x(0) \in U_{\delta} \Longrightarrow x(t) \in U_{\epsilon}, \forall t \geq 0$.

- asymptotically stable if it is stable and $\delta$ can be chosen such that $\lim _{t \rightarrow \infty} \operatorname{dist}\left(x(t), \mathcal{O}_{d}\right)=0$.

The control $u_{c}$ in (9) stabilizes $\mathcal{C}$ but does not stabilize $\mathcal{O}_{d}$. If $q, \dot{q} \in \mathcal{O}_{d}, u_{c}$ enforces the VHC and trajectories stay on $\mathcal{O}_{d}$; however, a perturbation of the states will cause the trajectories to converge to a different orbit on $\mathcal{C}$. The objective of this paper is to enforce the VHC and exponentially stabilize the desired orbit $\mathcal{O}_{d}$ on $\mathcal{C}$.

\footnotetext{
1 Assumptions 1 and 2 provide necessary and sufficient conditions for the reduced system to be Euler-Lagrange - the proof of this result can be found in $[14,16]$.
} 


\section{Main Result: Stabilization of $\mathcal{O}_{d}$}

\subsection{Poincaré Map}

The system in (1) with $u=u_{c}$ defined in (9), has the statespace representation

$$
\dot{x}=f(x)
$$

The stability characteristics of periodic orbits can be studied using Poincaré maps [24]. To this end, we define the Poincaré section $\Sigma$ of $\mathcal{O}_{d}$ as follow 2 :

$$
\Sigma=\left\{x \in \mathbb{Q}^{n} \times R^{n}: q_{2}=q_{2}^{*}, \dot{q}_{2} \geq 0\right\}
$$

where $q_{2}^{*}$ is a constant. Let $z, z \triangleq\left[q_{1}^{T}, \dot{q}^{T}\right]^{T} \in R^{(2 n-1)}$, denote the states of the system on $\Sigma$. The Poincare map $\mathbb{P}: \Sigma \rightarrow \Sigma$ is obtained by following trajectories of $z$ from one intersection with $\Sigma$ to the next. Let $t_{k}, k=1,2, \cdots$ denote the time of the $k$-th intersection and $z(k)=z\left(t_{k}\right)$. Then, $z(k+1)$ can be described with the help of the map $\mathbb{P}$

$$
z(k+1)=\mathbb{P}[z(k)]
$$

The point of intersection of $\Sigma$ and $\mathcal{O}_{d}$ is the fixed point of $\mathbb{P}$ denoted by $z^{*}$; it satisfies the following relation

$$
z^{*}=\mathbb{P}\left(z^{*}\right)
$$

The stability characteristics of the orbit $\mathcal{O}_{d}$ can be studied by investigating the stability properties of $z^{*}$, which is an equilibrium point of the discrete-time system in (17); this can be done by linearizing the map $\mathbb{P}$ about $z^{*}$. For $z(k)=$ $z^{*}+\nu$, where $\|\nu\|$ is a small number, we can write

$$
\begin{aligned}
z(k+1) & =\mathbb{P}\left(z^{*}+\nu\right) \\
& =\mathbb{P}\left(z^{*}\right)+\left[\nabla_{z} \mathbb{P}(z)\right]_{z=z^{*}}\left[z(k)-z^{*}\right]+O\left(\|\nu\|^{2}\right)
\end{aligned}
$$

Using $\mathbb{P}\left(z^{*}\right)=z^{*}$ from (18) and neglecting higher-order terms in $\|\nu\|$, the above equation can be written as

$$
\begin{gathered}
e(k+1)=\mathcal{A} e(k) \\
e(k) \triangleq z(k)-z^{*}, \quad \mathcal{A} \triangleq\left[\nabla_{z} \mathbb{P}(z)\right]_{z=z^{*}}
\end{gathered}
$$

The stability properties of $z^{*}$ is governed by the eigenvalues of $\mathcal{A}$, which are referred to as the Floquet multipliers of $\mathcal{O}_{d}$. If the Floquet multipliers lie inside the unit circle, $\mathcal{O}_{d}$ is exponentially stable - see Theorem 7.3 of [12]. From our discussion in section 2.4 we know that the desired orbit $\mathcal{O}_{d}$ is unstable, i.e., not all eigenvalues of $\mathcal{A}$ lie inside the unit circle. To stabilize the orbit, i.e., to stabilize $z^{*}$, we design an impulse controller in the next subsection.

\footnotetext{
${ }^{2}$ In the definition of $\Sigma$ in (16), $\dot{q}_{2} \geq 0$ can be replaced with
} $\dot{q}_{2} \leq 0$ without any loss of generality.

\subsection{Impulse Controlled Poincaré Map (ICPM)}

To stabilize the desired orbit $\mathcal{O}_{d}$, our controller is modified as follows

$$
u=u_{c}+u_{\mathcal{I}}
$$

where $u_{\mathcal{I}}$ is an impulsive input which is applied only when $x(t) \in \Sigma$. The dynamics of the system with $u_{\mathcal{I}}$ as the new input can be written as

$$
\begin{aligned}
& M_{11}(q) \ddot{q}_{1}+M_{12}(q) \ddot{q}_{2}+\bar{h}_{1}(q, \dot{q})=u_{\mathcal{I}} \\
& M_{12}^{T}(q) \ddot{q}_{1}+M_{22}(q) \ddot{q}_{2}+h_{2}(q, \dot{q})=0
\end{aligned}
$$

where $\bar{h}_{1} \triangleq\left(h_{1}-u_{c}\right)$. Impulsive inputs cause discontinuous changes in the generalized velocities while there is no change in the generalized coordinates. On the Poincaré section $\Sigma$, the jump in velocities can be computed by integrating (21) as follows [4]:

$$
\left[\begin{array}{ll}
M_{11} & M_{12} \\
M_{12}^{T} & M_{22}
\end{array}\right]\left[\begin{array}{c}
\Delta \dot{q}_{1} \\
\Delta \dot{q}_{2}
\end{array}\right]=\left[\begin{array}{c}
\mathcal{I} \\
0
\end{array}\right], \quad \mathcal{I} \triangleq \int_{0}^{\Delta t} u_{\mathcal{I}} d t
$$

In the above equation, $\Delta t$ is the infinitesimal interval of time for which $u_{\mathcal{I}}$ is active, $\mathcal{I} \in R^{n-1}$ is the impulse of the impulsive input, and $\Delta \dot{q}_{1}$ and $\Delta \dot{q}_{2}$ are defined as

$$
\Delta \dot{q}_{1} \triangleq\left(\dot{q}_{1}^{+}-\dot{q}_{1}^{-}\right), \quad \Delta \dot{q}_{2} \triangleq\left(\dot{q}_{2}^{+}-\dot{q}_{2}^{-}\right)
$$

where $\dot{q}^{-}$and $\dot{q}^{+}$are the velocities immediately before and after application of $u_{\mathcal{I}}$. Since the system is underactuated, the jump in the passive velocity $\dot{q}_{2}$ is dependent on the jumps in the active velocity $\dot{q}_{1}$; this relationship is described by the $(n-1)$ dimensional impulse manifold $[8,10]$, which can be obtained from (23):

$$
\mathcal{I}_{\mathrm{M}}=\left\{\dot{q}_{1}^{+}, \dot{q}_{2}^{+} \mid \Delta \dot{q}_{2}=-\left(1 / M_{22}\right) M_{12}^{T} \Delta \dot{q}_{1}\right\}
$$

Since impulsive inputs can cause the system states to move on $\Sigma$, we exploit this property to design a feedback law that stabilizes $z^{*}$, i.e., stabilizes $\mathcal{O}_{d}$. The control input applied at $t_{k}$ is denoted by $\mathcal{I}(k) 3$. The dynamics of the impulse controlled system in (17) can be described by the map

$$
z(k+1)=\mathbb{P}[z(k), \mathcal{I}(k)]
$$

where $\mathcal{I}(k)=0$ if $z(k)=z^{*}$. By linearizing the above map about the fixed point $z^{*}$ and $\mathcal{I}=0$, we get

$$
\begin{aligned}
e(k+1) & =\mathcal{A} e(k)+\mathcal{B} \mathcal{I}(k) \\
\mathcal{A} & \triangleq\left[\nabla_{z} \mathbb{P}(z, \mathcal{I})\right]_{z=z^{*}, \mathcal{I}=0} \\
\mathcal{B} & \triangleq\left[\nabla_{\mathcal{I}} \mathbb{P}(z, \mathcal{I})\right]_{z=z^{*}, \mathcal{I}=0}
\end{aligned}
$$

\footnotetext{
${ }^{3}$ As long as $\Delta t$ is sufficiently small, the effect of the impulsive input $u_{\mathcal{I}}$ depends solely on the value of $\mathcal{I}$ - see (23). Thus $\mathcal{I}$ can be viewed as the control input.
} 


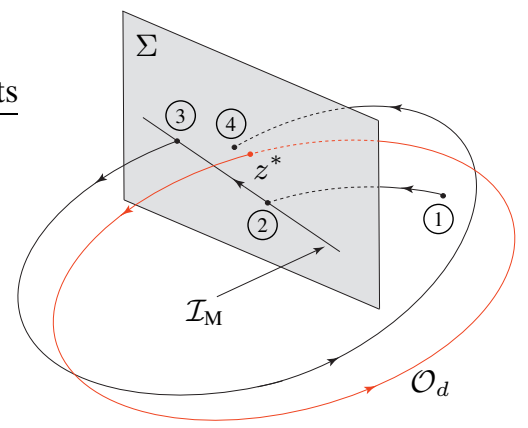

Fig. 1. Schematic of ICPM approach to orbital stabilization.

where $\mathcal{A} \in R^{(2 n-1) \times(2 n-1)}$ and $\mathcal{B} \in R^{(2 n-1) \times(n-1)}$ can be obtained numerically. Since $\mathcal{A}$ is not Hurwitz (see discussion in the last sub-section), we make the following proposition to stabilize $\mathcal{O}_{d}$ :

Proposition 1 If the pair $\{\mathcal{A}, \mathcal{B}\}$ is stabilizable, the orbit $\mathcal{O}_{d}$ can be stabilized using the discrete impulsive feedback

$$
\mathcal{I}(k)=\mathcal{K} e(k)
$$

where the matrix $\mathcal{K}$ is chosen such that $(\mathcal{A}+\mathcal{B} \mathcal{K})$ is Hurwitz.

The above approach to stabilization, which we refer to as the impulse controlled Poincaré map (ICPM) approach, is explained with the help of the schematic in Fig.1. The desired orbit $\mathcal{O}_{d}$ is shown in red and it intersects the Poincare section $\Sigma$ at the fixed point $z^{*}$. A trajectory starting from an arbitrary initial condition, shown by the point (1), intersects $\Sigma$ at (2). The impulsive input in (28) moves the configuration of the system from (2) to (3) along the impulse manifold $\mathcal{I}_{\mathrm{M}}$, where $\mathcal{I}_{\mathrm{M}} \subset \Sigma$. In other words, we impose the restriction that (3) lies on $\Sigma$, i.e., $\dot{q}_{2}^{+} \geq 0$. Hereafter, the altered system trajectory evolves under the continuous control $u_{c}$ and (4) denotes its next intersection with $\Sigma$. A series of ICPMs, similar to the map (2) $\rightarrow$ (4) exponentially converge the intersection point of the trajectory on $\Sigma$ to $z^{*}$.

\subsection{Implementation of Control Design}

\subsubsection{Numerical Computation of $\mathcal{A}$ and $\mathcal{B}$ matrices}

Let $\delta_{i}, i=1,2, \cdots, 2 n-1$, denote the $i$-th column of $\varepsilon_{1} I_{(2 n-1)}$, where $\varepsilon_{1}$ is a small number and $I_{(2 n-1)}$ is the identity matrix of size $(2 n-1)$. If $\mathcal{A}_{i}$ denotes the $i$-th column of $\mathcal{A}$, then $\mathcal{A}_{i}$ can be numerically computed as follows:

$$
\mathcal{A}_{i}=\frac{1}{\varepsilon_{1}}\left[\mathbb{P}\left(z^{*}+\delta_{i}\right)-z^{*}\right]
$$

Let $Q \in R^{n \times(n-1)}$ and $S \in R^{(n-1)}$ be defined as follows:

$$
Q \triangleq\left[\frac{I_{(n-1)}}{0_{1 \times(n-1)}}\right], \quad S \triangleq\left[\frac{0_{(n-1) \times 1}}{M(q)^{-1} \eta_{i}}\right]
$$

where $0_{i \times j}$ is a matrix of zeros of dimension $i \times j$, and $\eta_{i}$, $i=1,2, \cdots, n-1$, denote the $i$-th column of $\varepsilon_{2} Q$, where $\varepsilon_{2}$ is a small number. If $\mathcal{B}_{i}$ denotes the $i$-th column of $\mathcal{B}$, then $\mathcal{B}_{i}$ can be numerically computed as follows:

$$
\mathcal{B}_{i}=\frac{1}{\varepsilon_{2}}\left\{[\mathbb{P}(z+S)]_{z=z^{*}}-z^{*}\right\}
$$

The above expression has been obtained using (23).

\subsubsection{Impulsive Input using High-Gain Feedback}

Impulsive inputs are Dirac-delta functions and cannot be realized in real physical systems. Using singular perturbation theory [13], it was shown that continuous-time implementation of impulsive inputs can be carried out using highgain feedback [8]. To obtain the expression for the high-gain feedback, we substitute (28) in (23) to get

$$
\Delta \dot{q}_{1}(k)=B \mathcal{I}(k)=B \mathcal{K} e(k)
$$

where $B$ is defined in (3) and is evaluated at $t_{k} ; \Delta \dot{q}_{1}(k)$ is the jump in the active velocities generated by the input $\mathcal{I}(k)$. From (24) and (31), the desired active joint velocities at $t_{k}$ is

$$
\dot{q}_{1}^{\mathrm{des}}(k)=\dot{q}_{1}(k)+B \mathcal{K} e(k)
$$

where $\dot{q}_{1}(k)=\dot{q}_{1}\left(t_{k}\right)$. To reach the desired velocities in a very short period of time, we use the high-gain feedback [10]

$$
u_{\mathrm{hg}}=B^{-1}\left[\frac{1}{\mu} \Lambda\left(\dot{q}_{1}^{\mathrm{des}}(k)-\dot{q}_{1}\right)-\bar{A}\right]
$$

which remains active for as along as $\left\|\dot{q}_{1}^{\text {des }}(k)-\dot{q}_{1}\right\| \geq \varepsilon_{3}$, where $\varepsilon_{3}$ is a small number. In (33), $\dot{q}_{1}^{\text {des }}$ is obtained from (32) and $\bar{A}$ is obtained from the expression for $A$ in (3) by replacing $h_{1}$ with $\bar{h}_{1}$. Furthermore, $\Lambda \triangleq$ $\operatorname{diag}\left[\begin{array}{llll}\lambda_{1} & \lambda_{2} & \cdots & \lambda_{n-1}\end{array}\right]$, where $\lambda_{i}, i=1,2, \cdots, n-1$ are positive numbers, and $\mu>0$ is a small number.

\section{Illustrative Example: Cart-Pendulum}

\subsection{System Dynamics and VHC}

Consider the frictionless cart-pendulum system in Fig.2. The masses of the cart and pendulum are denoted by $m_{c}$ and $m_{p}$,

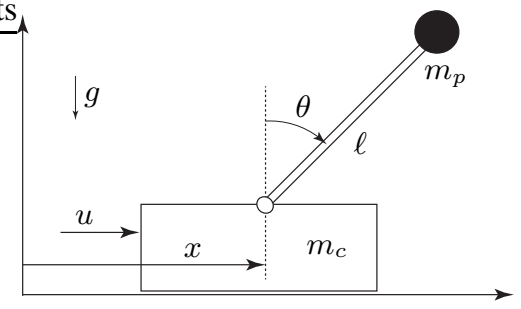

Fig. 2. Inverted pendulum on a cart. 
$\ell$ denotes the length of the pendulum, and $g$ is the acceleration due to gravity. The control input $u$ is the horizontal force applied on the cart. The cart position is denoted by $x$ and the angular displacement of the pendulum, measured clock-clockwise with respect to the vertical, is denoted by $\theta$. We consider physical parameters of the system to be the same as those in [20]: $m_{p}=m_{c}=\ell=1$. With the following definition

$$
q=\left[\begin{array}{ll}
q_{1} & q_{2}
\end{array}\right]^{T}=\left[\begin{array}{ll}
x & \theta
\end{array}\right]^{T}
$$

and the potential energy of the system, given by

$$
\mathcal{F}=\cos \theta
$$

the equations of motion can be obtained as

$$
\left[\begin{array}{cc}
2 & \cos \theta \\
\cos \theta & 1
\end{array}\right]\left[\begin{array}{l}
\ddot{x} \\
\ddot{\theta}
\end{array}\right]-\left[\begin{array}{c}
\sin \theta \dot{\theta}^{2} \\
g \sin \theta
\end{array}\right]=\left[\begin{array}{l}
u \\
0
\end{array}\right]
$$

which is of the form in (1). The VHC in (5) is chosen as

$$
\rho=x+1.5 \sin \theta=0
$$

which is identical to that considered in [20]. It can be verified that the mass matrix in (36) and the choice of VHC in (37) satisfy Assumptions 1 and 2 for $\bar{q}=(0,0)$. For the VHC in (37) to be stabilizable, Remark 1 provides the following condition that needs to be satisfied:

$$
1-1.5 \cos ^{2} \theta \neq 0 \Rightarrow \theta \neq \pm 0.61 \mathrm{rad}
$$

To compare our control design with that presented in [20], we assume that $\theta \in(-0.61,0.61)$ such that (38) is satisfied and the VHC in (37) is stabilizable. Simulation results will show that (38) is indeed satisfied.

\subsection{Stabilization of $\mathrm{VHC}$ and $\mathcal{O}_{d}$}

The ICPM approach relies on stabilization of both the constraint manifold $\mathcal{C}$, and the orbit $\mathcal{O}_{d}$ on $\mathcal{C}$. This is a distinctive difference between our approach and the approach in [20] where $\mathcal{O}_{d}$ is stabilized without stabilizing $\mathcal{C}$, i.e., without enforcing the VHC. To enforce the VHC, we choose the gains $k_{p}$ and $k_{d}$ in (9) as follows:

$$
k_{p}=2, \quad k_{d}=1
$$

We choose the desired orbit $\mathcal{O}_{d}$ to pass through the point:

$$
(x, \theta, \dot{x}, \dot{\theta})=(0.0,0.0,-0.675,0.450)
$$

which is approximately the desired orbit in [20] - see Fig.2 therein. To stabilize $\mathcal{O}_{d}$, we define the Poinacaré section

$$
\Sigma=\left\{x \in \mathbb{Q}^{2} \times R^{2}: \theta=0, \dot{\theta} \geq 0\right\}
$$

The states of the system on $\Sigma$ are

$$
z=\left[\begin{array}{lll}
x & \dot{x} & \dot{\theta}
\end{array}\right]^{T}
$$

Since $z^{*}$ lies on $\mathcal{O}_{d}$, using (40) and (41) we get

$$
z^{*}=\left[\begin{array}{lll}
0.0 & -0.675 & 0.450
\end{array}\right]^{T}
$$

The matrices $\mathcal{A}$ and $\mathcal{B}$ in (29) and (30) are obtained as

$$
\mathcal{A}=\left[\begin{array}{rrr}
0.115 & 0.435 & 0.600 \\
-0.510 & -0.640 & -2.465 \\
-0.145 & 0.215 & 1.325
\end{array}\right], \quad \mathcal{B}=\left[\begin{array}{r}
-0.06 \\
1.80 \\
-1.09
\end{array}\right]
$$

It can be verified that the eigenvalues of $\mathcal{A}$ do not lie inside the unit circle but the pair $\{\mathcal{A}, \mathcal{B}\}$ is controllable and satisfy Proposition 1. Using LQR design, the gain matrix $\mathcal{K}$ in (28) was obtained as

$$
\mathcal{K}=\left[\begin{array}{lll}
0.163 & 0.288 & 1.198
\end{array}\right]
$$

The eigenvalues of $(\mathcal{A}+\mathcal{B K})$ are located at 0.13 and $-0.06 \pm$ $0.48 i$; this implies that the impulsive feedback exponentially stabilizes the desired orbit $\mathcal{O}_{d}$.

\subsection{Simulation Results}

The initial configuration of the system is taken from [20]:

$$
\left[\begin{array}{llll}
x & \theta & \dot{x} & \dot{\theta}
\end{array}\right]=\left[\begin{array}{llll}
0.1 & 0.4 & -0.1 & -0.2
\end{array}\right]
$$

For the controller gains in (39) and (42), simulation results for the ICPM are shown in Fig.3; $\rho$ is plotted with time in Fig.3 (a) and the phase portrait of the pendulum is shown in Fig.3 (b). It can be seen from Fig.3 (a) that the continuous controller $u_{c}$ in (9) enforces the VHC in (37). To stabilize $\mathcal{O}_{d}$, the impulsive controller in (28) is implemented using the high-gain feedback in (33) with $\Lambda=1$ and $\mu=0.005$. It can be seen from the phase portrait in Fig.3 (b) that the pendulum trajectory converges exponentially to $\mathcal{O}_{d}$, shown in red. The effect of discrete impulsive feedback can be
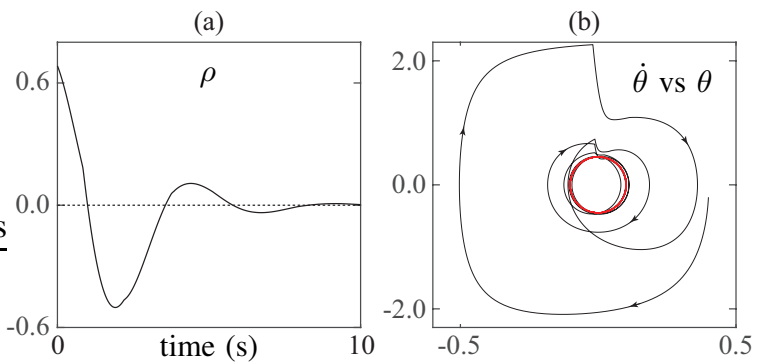

Fig. 3. Orbital stabilization for the cart-pendulum system; the initial conditions were taken from [20]. 

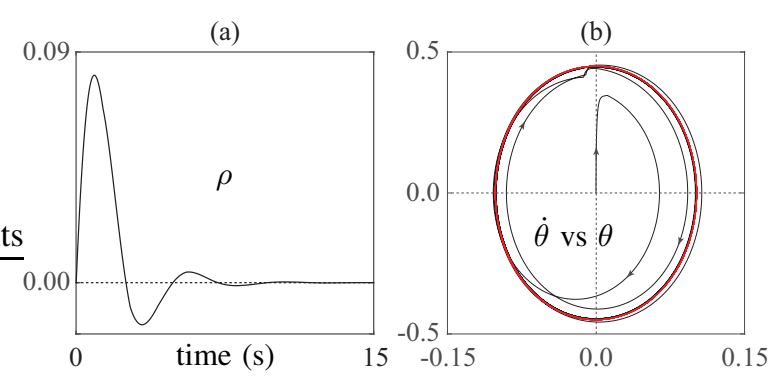

Fig. 4. Orbital stabilization for the cart-pendulum system for the initial conditions in (43).

seen in Fig.3 (b) where $\dot{\theta}$ jumps when trajectories cross the Poincaré section $\Sigma$ defined in (41). The system trajectories reach a close neighborhood of $\mathcal{O}_{d}$ in approximately $10 \mathrm{sec}$; this is comparable to the results in [20].

We now consider the following initial condition that lies far away from $\mathcal{O}_{d}$ :

$$
\left[\begin{array}{llll}
x & \theta & \dot{x} & \dot{\theta}
\end{array}\right]=\left[\begin{array}{llll}
0.0 & 0.0 & 0.0 & 0.0
\end{array}\right]
$$

We used the same controller gains as that used in the previous simulation. It can be seen from the results shown in Fig.4 that $\mathcal{O}_{d}$ is stabilized. For the initial conditions in (43), the control design in [20] fails to converge the pendulum trajectory to $\mathcal{O}_{d}$; this implies that $\mathcal{O}_{d}$ has a larger region of attraction with the ICPM approach than with the control design in [20]. To demonstrate the generality of the ICPM approach, we consider the three DOF tiptoebot, which is presented next.

\section{Illustrative Example - The Tiptoebot}

\subsection{System Description}

Consider the three DOF tiptoebot [10] shown in Fig.5. The tiptoebot is a human-like underactuated system with one passive joint; the three links are analogous to the lower leg, upper leg and torso. The knee joint connecting the upper and

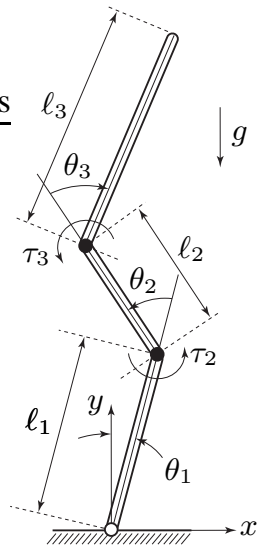

Fig. 5. The three-link underactuated tiptoebot. lower legs, and the hip joint connecting the torso and upper leg are active; the torques applied by the actuators in these joints are assumed to be positive in the counter-clockwise direction and are denoted by $\tau_{2}$ and $\tau_{3}$. The toe provides a point of support and is a passive revolute joint. The joint angles of the links, $\theta_{1}, \theta_{2}$ and $\theta_{3}$, are measured positive in the counter-clockwise direction; $\theta_{1}$ is measured relative to the $y$-axis and $\theta_{2}$ and $\theta_{3}$ are measured relative to the first and second links. Using the following definition for the joint angles and control inputs

$$
q_{1}^{T}=\left[\begin{array}{ll}
\theta_{2} & \theta_{3}
\end{array}\right]^{T}, \quad q_{2}=\theta_{1}, \quad u=\left[\begin{array}{ll}
\tau_{2} & \tau_{3}
\end{array}\right]^{T}
$$

the dynamics of the tiptoebot can be expressed in the form given in (1), where the components of the mass matrix and the potential energy are:

$$
\begin{aligned}
& M_{11}=\left[\begin{array}{cc}
\alpha_{2}+\alpha_{3}+2 \alpha_{5} \cos \theta_{3} & \alpha_{3}+\alpha_{5} \cos \theta_{3} \\
\alpha_{3}+\alpha_{5} \cos \theta_{3} & \alpha_{3}
\end{array}\right] \\
& M_{12}=\left[\begin{array}{c}
\alpha_{2}+\alpha_{3}+\alpha_{4} \cos \theta_{2}+2 \alpha_{5} \cos \theta_{3}+\alpha_{6} \cos \left(\theta_{2}+\theta_{3}\right) \\
\alpha_{3}+\alpha_{5} \cos \theta_{3}+\alpha_{6} \cos \left(\theta_{2}+\theta_{3}\right)
\end{array}\right] \\
& M_{22}=\alpha_{1}+\alpha_{2}+\alpha_{3} \\
&+2\left[\alpha_{4} \cos \theta_{2}+\alpha_{5} \cos \theta_{3}+\alpha_{6} \cos \left(\theta_{2}+\theta_{3}\right)\right] \\
& \mathcal{F}=\beta_{1} \cos \theta_{1}+\beta_{2} \cos \left(\theta_{1}+\theta_{2}\right)+\beta_{3} \cos \left(\theta_{1}+\theta_{2}+\theta_{3}\right)
\end{aligned}
$$

where $\alpha_{i}, i=1,2, \cdots, 6$, and $\beta_{i}, i=1,2,3$ are lumped physical parameters; their values are given in Table 1. It can be verified that Assumption 1 is satisfied for $\bar{q}=\left(\begin{array}{lll}0 & 0 & 0\end{array}\right)^{T}$.

Table 1: Tiptoebot lumped parameters in SI units

\begin{tabular}{|l|l|l|l|l|l|}
\hline$\alpha_{1}$ & 0.386 & $\alpha_{4}$ & 0.065 & $\beta_{1}$ & 4.307 \\
\hline$\alpha_{2}$ & 0.217 & $\alpha_{5}$ & 0.054 & $\beta_{2}$ & 1.102 \\
\hline$\alpha_{3}$ & 0.247 & $\alpha_{6}$ & 0.104 & $\beta_{3}$ & 1.764 \\
\hline
\end{tabular}

\subsection{Imposing $\mathrm{VHC}$ and Selection of $\mathcal{O}_{d}$}

The VHC in (5) is chosen as

$$
\rho=\left[\begin{array}{l}
\rho_{1} \\
\rho_{2}
\end{array}\right]=\left[\begin{array}{l}
\theta_{2}-A_{1} \theta_{1} \\
\theta_{3}-A_{2} \theta_{1}
\end{array}\right]=\left[\begin{array}{l}
0 \\
0
\end{array}\right]
$$

where $A_{1}=-2$ and $A_{2}=0.1$. It can be verified that the VHC in (46) satisfies Assumption 2 for $\bar{q}=\left(\begin{array}{lll}0 & 0 & 0\end{array}\right)^{T}$; also, it is stabilizable as it satisfies the condition in Remark 1. To enforce the VHC, the gain matrices in (9) were chosen as

$$
k_{p}=\left[\begin{array}{ll}
1.0 & 0.0 \\
0.0 & 1.0
\end{array}\right], \quad k_{d}=\left[\begin{array}{ll}
0.1 & 0.0 \\
0.0 & 0.1
\end{array}\right]
$$

The phase portrait of the zero dynamics in (12) is shown in Fig.6. It can be seen that the equilibrium $\left(\theta_{1}, \dot{\theta}_{1}\right)=(0,0)$ 
is a center, surrounded by a dense set of closed orbits. We choose the desired orbit $\mathcal{O}_{d}$ to be the one that passes through $\left(\theta_{1}, \dot{\theta}_{1}\right)=(0.0,3.0)$.

\subsection{Stabilization of $\mathcal{O}_{d}$}

The desired orbit $\mathcal{O}_{d}$, shown in red in Fig.6, is symmetric about $\theta_{1}=0$ and without loss of generality we define the Poincaré section of $\mathcal{O}_{d}$ as follows

$$
\Sigma=\left\{x \in \mathbb{Q}^{3} \times R^{3}: \theta_{1}=0, \dot{\theta}_{1} \geq 0\right\}
$$

The states on $\Sigma$ are

$$
z=\left[\begin{array}{lllll}
\theta_{2} & \theta_{3} & \dot{\theta}_{1} & \dot{\theta}_{2} & \dot{\theta}_{3}
\end{array}\right]^{T}
$$

The fixed point $z=z^{*}$ lies on $\mathcal{O}_{d}$ and satisfies the VHC relationship $\rho=\dot{\rho}=0$. Substituting $\left(\theta_{1}, \dot{\theta}_{1}\right)=(0.0,3.0)$ in (46) and its derivative gives

$$
z^{*}=\left[\begin{array}{lllll}
0.0 & 0.0 & 3.0 & -6.0 & 0.3
\end{array}\right]^{T}
$$

The matrices $\mathcal{A}$ and $\mathcal{B}$ in (29) and (30) were obtained as

$$
\begin{aligned}
& \mathcal{A}=\left[\begin{array}{rrrrr}
-0.380 & -0.080 & 1.530 & 0.800 & 0.050 \\
0.000 & -0.460 & -0.080 & -0.003 & 0.730 \\
1.230 & 1.890 & 6.120 & 2.770 & 4.050 \\
-3.210 & -3.770 & -13.360 & -6.090 & -8.100 \\
0.120 & -0.560 & 0.670 & 0.280 & 0.100
\end{array}\right] \\
& \mathcal{B}=\left[\begin{array}{rrrrr}
1.525 & -3.700 & -17.700 & 34.325 & 0.875 \\
4.875 & -8.650 & 22.650 & -43.850 & -0.325
\end{array}\right]^{T}
\end{aligned}
$$

The eigenvalues of $\mathcal{A}$ do not lie inside the unit circle but the pair $\{\mathcal{A}, \mathcal{B}\}$ is stabilizable and satisfy Proposition 1 . Using $\mathrm{LQR}$, the gain matrix $\mathcal{K}$ in (28) is obtained as

$$
\mathcal{K}=\left[\begin{array}{rrrrr}
0.028 & 0.024 & 0.197 & 0.094 & 0.138 \\
-0.034 & -0.051 & 0.116 & -0.049 & -0.055
\end{array}\right]
$$

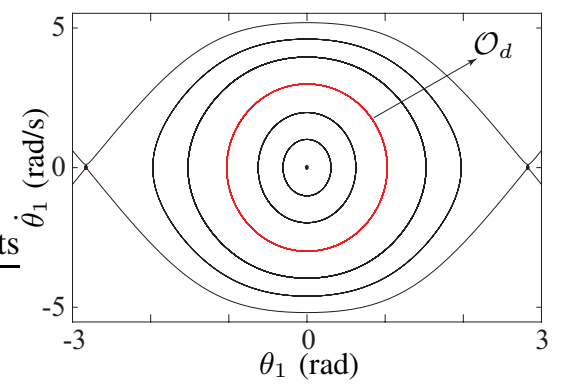

Fig. 6. Phase portrait of tiptoebot zero dynamics.
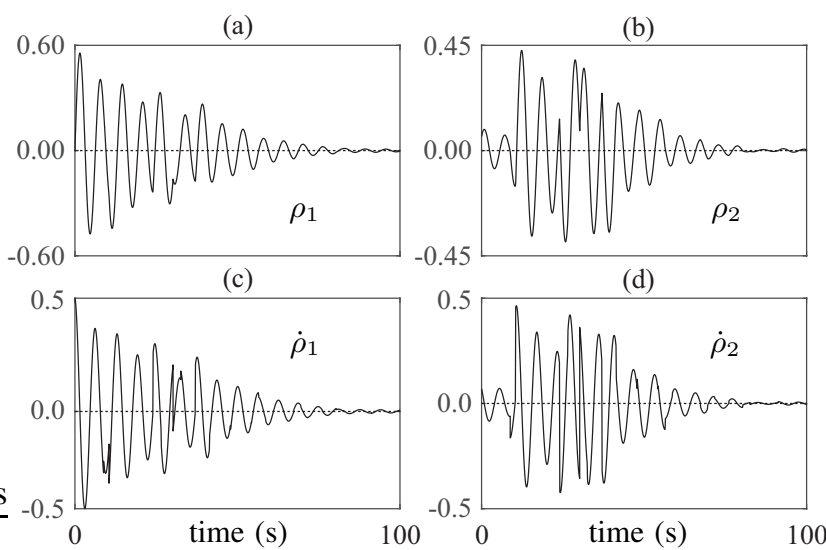

(e)

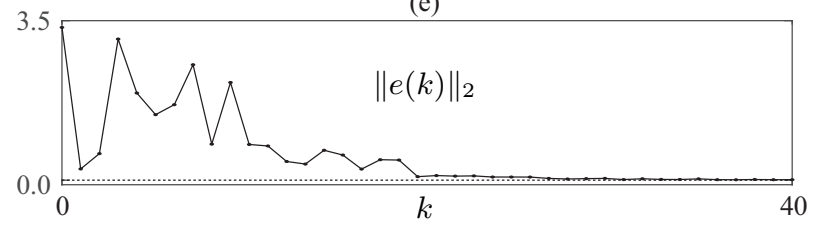

Fig. 7. Orbital stabilization for the tiptoebot using ICPM.

The eigenvalues of $(\mathcal{A}+\mathcal{B K})$ are located at $0.14,-0.47 \pm$ $0.73 i$ and $-0.12 \pm 0.56 i$; this implies that $\mathcal{O}_{d}$ is exponentially stable.

\subsection{Simulation Results}

The initial configuration of the tiptoebot is taken as

$$
\left[\begin{array}{llllll}
\theta_{1} & \theta_{2} & \theta_{3} & \dot{\theta}_{1} & \dot{\theta}_{2} & \dot{\theta}_{3}
\end{array}\right]=\left[\begin{array}{llllll}
-0.1 & 0.2 & 0.05 & 3.3 & -6.0 & 0.4
\end{array}\right]
$$

For the controller gains in (47) and (50), simulation results of the ICPM approach are shown in Fig.7. The plots of $\rho_{1}$, $\rho_{2}, \dot{\rho}_{1}$ and $\dot{\rho}_{2}$ with time are shown in Figs.7 (a)-(d); it can be seen that the continuous controller $u_{c}$ in (9) enforces the VHC in (46) by stabilizing the constraint manifold $\mathcal{C}$. To stabilize the desired orbit $\mathcal{O}_{d}$, the impulsive controller in (28) is implemented using the high-gain feedback in (33); $\Lambda$ was chosen to be an identity matrix and $\mu$ was chosen as 0.0001 . To show the convergence of system trajectories to $\mathcal{O}_{d},\|e(k)\|_{2}$ is plotted with respect to $k$ in Fig.7 (e). It can be seen that for large values of $k,\|e(k)\|_{2} \rightarrow 0$; this implies that $\mathcal{O}_{d}$ is exponentially stable.

\section{Conclusion}

Repetitive motion in underactuated systems are typically designed using VHCs. A VHC results in a family of periodic orbits and stabilization of an orbit is an important problem in applications such as legged locomotion. A hybrid control design was presented to stabilize a VHC-generated periodic orbit for underactuated system with one passive DOF; a continuous controller was used to enforce the VHC and impulsive inputs were periodically applied on a Poincaré section to stabilize the desired orbit. These impulsive inputs 
alter the Poincaré map and this impulse controlled Poincaré map (ICPM) is described by a discrete time-invariant linear system. The problem of orbital stabilization problem is thus simplified to stabilization of the fixed point of the ICPM. The controllability of the system can be easily verified and the control design can be easily carried out using standard techniques such as pole-placement and LQR. The identification of the linear system and computation of the controller gains are performed off-line. The complexity and computational cost of the ICPM approach is less than existing methods in the literature as it eliminates the need for on-line solution of a periodic Ricatti equation. The ICPM approach is demonstrated using the standard cart-pendulum system; its applicability to higher-dimensional systems is demonstrated using the three-DOF tiptoebot. Future work will focus on gait stabilization of legged robots undergoing ground-foot impacts and experimental validation.

\section{References}

[1] Thamer Albahkali, Ranjan Mukherjee, and Tuhin Das. Swing-up control of the pendubot: An impulse-momentum approach. IEEE Trans. on Robotics, 25(4):975-982, 2009.

[2] Carlos Canudas-de Wit. On the concept of virtual constraints as a tool for walking robot control and balancing. Annual Reviews in Control, 28(2):157-166, 2004.

[3] Carlos Canudas-de Wit, Bernard Espiau, and Claudio Urrea. Orbital stabilization of underactuated mechanical systems. In Proc. 15th IFAC World Congress, 2002.

[4] Louis L Flynn, Rouhollah Jafari, and Ranjan Mukherjee. Active synthetic-wheel biped with torso. IEEE Trans. on Robotics, 26(5):816-826, 2010

[5] Leonid Freidovich, Anders Robertsson, Anton Shiriaev, and Rolf Johansson. Periodic motions of the pendubot via virtual holonomic constraints: Theory and experiments. Automatica, 44(3):785-791, 2008.

[6] Jessy W Grizzle, Gabriel Abba, and Franck Plestan. Asymptotically stable walking for biped robots: Analysis via systems with impulse effects. IEEE Trans. on Automatic Control, 46(1):51-64, 2001.

[7] Jessy W Grizzle, Christine Chevallereau, Ryan W Sinnet, and Aaron D Ames. Models, feedback control, and open problems of 3d bipedal robotic walking. Automatica, 50(8):1955-1988, 2014.

[8] Rouhollah Jafari, Frank B Mathis, Ranjan Mukherjee, and Hassan Khalil. Enlarging the region of attraction of equilibria of underactuated systems using impulsive inputs. IEEE Trans. on Control Systems Technology, 24(1):334-340, 2016.

[9] Nilay Kant and Ranjan Mukherjee. Impulsive dynamics and control of the inertia-wheel pendulum. IEEE Robotics and Automation Letters, 3(4):3208-3215, 2018.
[10] Nilay Kant, Ranjan Mukherjee, Dhrubajit Chowdhury, and Hassan K Khalil. Estimation of the region of attraction of underactuated systems and its enlargement using impulsive inputs. IEEE Trans. on Robotics, 35(3):618-632, 2019.

[11] Nilay Kant, Ranjan Mukherjee, and Hassan K Khalil. Stabilization of homoclinic orbits of two degree-of-freedom underactuated systems. In Proc. American Control Conference, 2019.

[12] Hassan K Khalil. Nonlinear Systems. Prentice Hall Upper Saddle River, NJ, second edition, 1996.

[13] Petar Kokotovic, Hassan K Khalil, and John O'Reilly. Singular perturbation methods in control: Analysis and design, volume 25. Siam, 1999.

[14] Manfredi Maggiore and Luca Consolini. Virtual holonomic constraints for Euler-Lagrange systems. IEEE Trans. on Automatic Control, 58(4):1001-1008, 2012.

[15] Frank B Mathis, Rouhollah Jafari, and Ranjan Mukherjee. Impulsive actuation in robot manipulators: Experimental verification of pendubot swing-up. IEEE/ASME Trans. on Mechatronics, 19(4):1469-1474, 2014.

[16] Alireza Mohammadi. Virtual holonomic constraints for EulerLagrange control systems. $\mathrm{PhD}$ thesis, University of Toronto (Canada), 2016.

[17] Alireza Mohammadi, Manfredi Maggiore, and Luca Consolini. Dynamic virtual holonomic constraints for stabilization of closed orbits in underactuated mechanical systems. Automatica, 94:112124, 2018.

[18] Alireza Mohammadi, Ehsan Rezapour, Manfredi Maggiore, and Kristin Y Pettersen. Maneuvering control of planar snake robots using virtual holonomic constraints. IEEE Trans. on Control Systems Technology, 24(3):884-899, 2015.

[19] N. Nekhoroshev. The Poincaré-Lyapunov-Liouville-Arnol'd theorem. Functional Analysis and Its Applications, 28:128-129, 041994.

[20] Anton Shiriaev, John W Perram, and Carlos Canudas-de Wit. Constructive tool for orbital stabilization of underactuated nonlinear systems: Virtual constraints approach. IEEE Trans. on Automatic Control, 50(8):1164-1176, 2005.

[21] Anton Shiriaev, Anders Robertsson, John Perram, and Anders Sandberg. Periodic motion planning for virtually constrained EulerLagrange systems. Systems \& Control Letters, 55(11):900-907, 2006.

[22] Anton S Shiriaev, Leonid B Freidovich, and Sergei V Gusev. Transverse linearization for controlled mechanical systems with several passive degrees of freedom. IEEE Trans. on Automatic Control, 55(4):893-906, 2010.

[23] Anton S Shiriaev, Leonid B Freidovich, Anders Robertsson, Rolf Johansson, and Anders Sandberg. Virtual-holonomic-constraintsbased design of stable oscillations of Furuta pendulum: Theory and experiments. IEEE Trans. on Robotics, 23(4):827-832, 2007.

[24] Steven H Strogatz. Nonlinear dynamics and chaos: With applications to physics, biology, chemistry, and engineering. CRC press, 2018.

[25] Eric R Westervelt, Jessy W Grizzle, Christine Chevallereau, Jun Ho Choi, and Benjamin Morris. Feedback control of dynamic bipedal robot locomotion. CRC press, 2018. 